Meta

Journal des tradlucteurs

Translators' Journal

\title{
L'oeuvre en traduction
}

\section{Roland Houde}

Volume 23, numéro 3, septembre 1978

URI : https://id.erudit.org/iderudit/003139ar

DOI : https://doi.org/10.7202/003139ar

Aller au sommaire du numéro

Éditeur(s)

Les Presses de l'Université de Montréal

ISSN

0026-0452 (imprimé)

1492-1421 (numérique)

Découvrir la revue

Citer cet article

Houde, R. (1978). L'oeuvre en traduction. Meta, 23(3), 220-225.

https://doi.org/10.7202/003139ar

Ce document est protégé par la loi sur le droit d'auteur. L’utilisation des services d'Érudit (y compris la reproduction) est assujettie à sa politique d'utilisation que vous pouvez consulter en ligne.

https://apropos.erudit.org/fr/usagers/politique-dutilisation/
Cet article est diffusé et préservé par Érudit.

Érudit est un consortium interuniversitaire sans but lucratif composé de l’Université de Montréal, l'Université Laval et l'Université du Québec à Montréal. Il a pour mission la promotion et la valorisation de la recherche. https://www.erudit.org/fr/ 


\title{
L'œuvre en traduction
}

\begin{abstract}
«Les livres sont l'œuvre de la solitude et les enfants du silence. Les enfants du silence ne doivent rien avoir de commun avec les enfants de la parole» (Proust).
\end{abstract}

En 1944, l'abbé Arthur Maheux, O.B.E., dans un livre au titre toujours actuel (Problems of Canadian Unity, Québec, les Editions des Bois-Francs, p. 63-64), proposait un projet national mais d'envergure normale pour les intellectuels canadiens et les institutions concernées:

...there is another field that has hardly been cultivated, I mean the field of translation.

There are many Canadian Books, there are French and English books that should be translated. Canada is in the best position for a work of that sort.

Presently the public at large is deprived of real good reading because there are practically no translations.

This came to my mind last Sunday, when Lady Fiset, the Châtelaine of Spencer Wood asked me if I would translate Miss Elliott's very good book, Women Pioneers of North America. I feel sure that the French Canadians would appreciate Miss Elliott's wonderful narrative in their own language. The Association of Canadian Authors and the Société des Ecrivains canadiens might organise a joint Committee for the selection of the best books in both languages and for having them translated. I think it would mean much for a better understanding between Canadians.

L'année précédente, cet historien de l'Université Laval avait posé la question en français puis en anglais: Pourquoi sommes-nous divisés? (Montréal, Radio-Canada, 1943, 219 p., ou What Keeps Us Apart, Québec, 1944, 186 p.). Un autre abbé, Lionel Groulx, répondit rapidement et brièvement dans un tract: Pourquoi nous sommes divisés (Montréal, Action nationale, 1943, 30 p.), qui fut aussitôt traduit par le professeur Rothney de Sir George William College: Why We are Divided, A Reply $(1943,32$ p. avec introduction).

$\mathrm{Ne}$ faut-il pas se souvenir également qu'il existait alors une Société des traducteurs à Montréal ainsi qu'une Association technologique de langue française à Ottawa regroupant d'une façon ou d'une autre les interprètes bilingues du temps comme Jeannine Bélanger, Pierre Benoit, Ernest Bilodeau, Etienne Blanchard (18831952), Arthur Beauchesne (1873-1956), Georges Bouchard, Lucien Brault, André Champoux, Robert Choquette, Pierre Daviault, Jean-Louis Gagnon, Gérard Gardner, Léon Gérin, Jean-Charles Harvey, Gustave Lanctôt, Guillaume Lavallée, Georges Langlois, Maxine (pseudo. de Mme François-Elzéard-Achille TaschereauFortier, née Marie Caroline-Alexandre Bouchette), Paul-Maurice Olliver, Gérard Petit, Robert Prévost, H.-S. Saint-Denis, Georges Simard, Guy Sylvestre, Thérèse 
Tardif, Louvigny de Montigny et j'en passe parmi les mieux connus, par exemple, Edmond Robillard, o.p., m.a.c.f., s.o.b., Tous dans la foulée exemplaire d'autres traducteurs: d'un Ls.G. Gladu qui a traduit Thomas d'Arcy McGee, imprimé sur les Presses à Pouvoir du Courrier de St-Hyacinthe en 1865, d'un Louis-Ph. Geoffrion, d'un Arthur Buies, d'un Aegidius Fauteux (1876-1941), d'un Benjamin Sulte qui a rendu accessible The Story of Our Country de J.C. Hopkins en Histoire populaire du Canada (T. Winston, 1901, XVI-698 p.), ou d'un Jacques Labrie et les Premiers Rudiments de la Constitution britannique (trad. de l'anglais de M. Brooke, précédés d'un précis..., Montréal, James Lane, 1827, XI-89 p. avec "erratum»).

Depuis ce temps, le progrès s'est imposé. Nous avons maintenant des Écoles de traduction... gouvernementale et commerciale. Il existe de plus un comité de la traduction au Conseil canadien de recherches sur les humanités (CCRH/HRCC, 151 Slater, Ottawa). Ce comité a fait préparer par les soins du professeur Philip Stratford de l'Université de Montréal une Bibliographie de livres canadiens traduits de l'anglais au français et du français à l'anglais / Bibliography of Canadian Books in Translation: French to English and English to French. Une première édition parut en 1975, puis une deuxième augmentée, tout récemment: (xvii-78 p., avec index des traducteurs et des auteurs; 640 titres ou références; $\$ 1$ ). A ce prix, qui aurait quelque réticence à se la procurer?

De quoi s'agit-il? D'un répertoire sommaire, d'un inventaire préliminaire avec une préface du compilateur centrée sur l'évolution de la traduction littéraire au Canada. Par traduction et tradition littéraires, le professeur Stratford entend les genres littéraires ou catégories suivantes: roman, poésie, théàtre (sic), lettres rapports - relations de voyage, essais, anthologies, livres pour enfants, folklore, religion. Mais dès l'abord, ne faut-il pas remarquer que les propos liminaires du compilateur ne sont pas supportés par des statistiques canadiennes très précises, par exemple combien de volumes furent enregistrés au ministère fédéral de l'Agriculture conformément à l'ancienne loi ? ou déposés à la Bibliothèque nationale d'Ottawa depuis 1950 et à la Bibliothèque nationale du Québec depuis 1967 selon les nouvelles lois? Et traduits par après ou simultanément? Le compilateur devrait, il nous semble, s'appuyer sur l'inventaire des catalogues de libraires canadiens, régionaux ou nationaux. Sur l'histoire de l'imprimerie nationale ou régionale, des presses commerciales ou universitaires. Mais il faut bien le reconnaitre, la détermination et l'orientation de l'ouvrage est tout autre. De fait, l'histoire de l'imprimerie d'Aegidius Fauteux, dans ses versions anglaise (1930) et française (1957), n'y trouve pas sa place.

Prenons donc le titre du travail à la lettre. Et demandons-nous ce qu'est un livre canadien... traduit? Ouvrage d'un auteur canadien? D'un éditeur canadien? D'un traducteur canadien? D'un Canadien canadien. Mais il y a plus encore. Car il y a des thèmes canadiens, des sources canadiennes, des sujets canadiens exploités ici ou ailleurs, normalement et librement. Depuis les premières "relations", les premiers voyages édités ailleurs, et pour cause, avant l'avènement $\mathrm{du}$ caractère canadien, français ou anglais ... et plus tard juif, allemand, slovaque, ukrainien. Mais de ce point de vue, on peut aussitôt remarquer l'absence de l'Histoire véritable et naturelle... de Pierre Boucher traduite par E.L. Montizambert (Montréal, 1883). D'autres plus malins remarqueront l'absence du Huron à la recherche de l'art, traduit du québécois par l'auteur, Gérald Robitaille, publié à Paris chez Losfeld, avec préface signée Henry 
Miller. Ou l'absence de l'Acadia ... d'Édouard Richard, traduit par amour et à compte d'auteur par Henri d'Arles (Québec-Boston, 1916-1921, 3 tomes). Pour ne rien dire de la pléthore de productions du genre "vocabulaire": Vocabulaire technique des bibliothécaires, bibliophiles et bibliographes (trad. Juliette Chabot, 1943); Léon Gérin, Vocabulaire pratique de l'anglais au français... (Montréal, 1937, 302 p.); L.H. Tremblay, The Pronunciation of the French Language on a Mechanical Principle (Québec, 1881); L.F. Gouin, A New System of French and English Pronunciation with or without a Master (Three Rivers, $1859,213 \mathrm{p}$. avec texte français); J.-B. Meilleur, Nouvelle Grammaire anglaise, rédigée d'après les meilleurs auteurs (St-Charles, Village Debartzch, 1833) et $A$ Treatise of the Pronunciation of the French Language or A Synopsis of Rules for Pronouncing the French Language (2nd ed., Montréal, 1841). Pour ne rien dire des essais généraux, tous aussi généreux, plus modernes: Mortimer Adler, How to Read a Book, traduit et publié par Belisle à Québec; l'importante traduction par Gilles Lane de Austin (How to Do Things With Words) : Quand dire, c'est faire (Paris, Ed. du Seuil, 1970); un autre exemple élargi de la coopération intellectuelle franco-canado-américaine serait: M.-D. Chenu, o.p., Towards Understanding Saint Thomas, o.p. (Chicago, Regnery, 1964, X-386 p.); de facture différente à tous égards: la mauvaise traduction "ursuline" de la pauvre Logic... de Vincent Edward Smith; et cette "somme" de Maritain, maintenant accessible au public anglais grâce à Gerald B. Phelan, Ralph MacDonald, Prof., and Mrs. Lawrence E. Lynch, Mrs. Alfred Byrne: Distinguish to Unite or The Degrees of Knowledge (New Y ork-London, Scribner's-Bles, 1959, xix-476 p.); également traduit par Phelan de Toronto: R. Maritain, The Prince of this World (The Institute of Mediaeval Studies, 1933) avec une 2e éd., en Angleterre (St-Dominic's Press) en 1936; et en 1948, cette autre traduction par Lewis Galantière et Phelan de J. Maritain: Existence and the Existent (New York, Pantheon Books avec réimpression chez Doubleday en 1957).

Considérons donc qu'il y a traductions et traductions, répertoires et répertoires. Le petit recueil qui nous concerne mérite bien une bonne aération mentale. Sans pour autant discourir sur les conditions ou difficultés d'une littérature coloniale ! Quelles en sont (ou seront) les normes d'inclusion ou d'exclusion? Jusqu'à plus amplement informé sur ces questions de détails, libre à nous d'appuyer surtout sur l'imprimé canadien, sur l'impression canadienne. Alors nous aimerions voir signaler cette très belle production: André Maurois, A Voyage to the Island of the Articoles (trad. by David Granett, Montréal, Louis Carrier, At the Mercury, 1929). Et cette autre: Emery Reves, Manifeste démocratique (trad. Maurice-Edgar Coindreau, Montréal, Beauchemin, 1944). Sans oublier pourtant qu'il y aura toujours cette liberté foncière de l'acte créateur nécessaire et antérieure à l'art du traducteur conjuguant avec la décision finale reliée plus ou moins avec les rapports propres ou impropres de «comités» de lecture. Aventures conjointes ou disjointes ? Continues ou discontinues? En répondant à ces questions, le lecteur canadien sera alors peut-être en meilleure posture pour pouvoir apprécier toute l'histoire ou toute la genèse littéraire (humaine) d'un imprimé, d'une adaptation, d'une traduction, d'une relation auteurtraducteur-imprimeur-lecteur. Pour apprécier à sa juste valeur la longue relation entre Wm. Henry Drummond et Louis Fréchette de 1897 à 1923 ! Entre J.-Ch. Falardeau et E.C. Hughes, ou la rencontre de la sociologie québécoise et américaine qui s'enregistre dès 1944 chez l'éditeur Lucien Parizeau à Montréal! Donc bien avant ce qu'on peut 
voir ou ne pas voir à la p. 47, item Hughes, dans le travail que nous avons sous les yeux. Que faire de J. Bruchési, A History of Canada (trad. Clarke, Irwin, 1950, 338 p.)? De Dominique de St-Denis, The Catholic Church in Canada (Montréal, Thian, 1956, 269 p.) ou l'Église catholique au Canada... (Montréal, Couvent des capucins)? Et de Bernard Lonergan? Et Borduas en anglais? En attendant la nouvelle édition de cette "bibliographie", à paraître dans deux ans, qu'on nous permette de faire quelques suggestions de correction: (p. 1), de Gaspé, P.A.; G.M. Pennée au lieu de Pené; (item Bessette), Paris au lieu de Parir ; (p. 5), (item Hémon), Mac Phail au lieu de Mc Phail; (p. 6), l'item Marie-Victorin demande une normalisation bibliographique; (p. 15), l'item Hémon demande également une révision, sinon comment expliquer une «Trans. » en 1924 d'un texte français publié en 1928?; (p. 20), l'item Garneau demande une révision de la tomaison des différentes éditions; dans la série $\mathrm{B}$. des traductions de l'anglais au français (p. $35 \mathrm{ss}$ ), pourquoi le «Trans. " ou «N. Trans. " plut ôt que Trad. ou Nouv. Trad.? (p. 36), item Graham, Champoux au lieu de Champroux; même correction, p. 63 et p. 66-67 respectivement, pour MacPhail et Pennée.

Répondant enfin à l'invitation qui nous est faite (p. IX), en reconnaissance du service rendu par le compilateur, nous proposons une liste d'additions en ordre alphabétique ainsi qu'un index supplémentaire des auteurs bilingues canadiens (imprimés canadiens anglais-français / français-anglais, mais ne serait-il pas suffisant de dire canadiens/canadian?) qui devraient éventuellement se retrouver dans un répertoire augmenté ou dans tout prolégomène à toute histoire future de la traduction canadienne fondée sur l'historiographie des traducteurs ou interprètes canadiens, dans toutes les disciplines littéraires du Canada.

Il faut bien finalement en convenir. Dans cette bibliographie choisie, les champs littéraires les plus négligés sont les biographies, la lexicographie canadienne (bilingue), les bibliographies et les essais sur l'éducation, la philosophie. Quant aux relations de voyages, elles commanderaient à elles seules une bibliographie spécifique.

\section{ROLAND HOUDE}

\section{ADDENDA}

ANGLIN, Gerald, le Canada illimité, trad. Placide Labelle, Toronto, 1948.

ARNOLD, Matthew, Etudes sur les États-Unis, trad. Léonce Rinfret et Edmond de Nevers, avec préf. et notes par E. de N., Québec, 1902.

BAF. BEAU, Marius, Ceinture fléchée, Montréal, 1946 (adapt. et trad. augmentée de Assomption Sash. Ottawa, 1939).

BARTLETT, William Henry, Québec 1800, un essai... A 19th Century Romantic Sketch of Quebec, Montréal, Ed. de l'Homme. 1968 (texte et présentation bilingue: Michel Brunet et Russell J. Harper de Canadian Scenery).

BER.NIER, J.E. (Capt.), Rapport sur la croisière faite par le 3-mats mixte Arctic... Ottawa, $1910 \times 1 \mathrm{vil}-573 \mathrm{p}$. Report on the Dominion of Canada Government Expedition to the Arctic Island... Ottawa, $1910 \times x$ ix $-529 \mathrm{p}$

CARTIER, Jacques, The Voyages of Jacques Cartier... with transl. and notes by H.P. Biggar, Ottawa, 1924 , textes francais et anglais, xiv-330 p., 16 planches et cartes.

BOURASSA, Henri, Grande-Bretagne et Canada... Montréal, 1901, trans. Great Britain and Canada... Montréal, 1902.

BOYD; John, Sir Geo.-Et. Cartier, baronet. Sa vie et son temps... trad. Sylva Clapin, Montréal, 1918, XXviii-485 p.

BRETON, P.N., Histoire illustrée des monnaies et jetons du Canada, Montréal, 1894, texte bilingue, $239 \mathrm{p}$. COMEAU, Napoléon-A., Ia Vie et le sport sur la Côte-Nord... trad. N. LeVasseur, Québec, $1945,372 \mathrm{p}$. 
CURWOOD, J. Oliver, Nomades du nord, trad. Louis Postif, préf. M. Constantin-Weyer, P. Mornay, 1932, v-275 p. [ Ne faut-il pas signaler de plus qu'en 1938 le jeune Nestor du Roi des Aulnes, de Michel Tournier (1976, coll. Folio, no. 656, p. 63-64) "connaissait par coeur des pages entières * du Piège d'Or de J.O.C. !]

DE CELLES, A.D., The Habitant, Toronto, 1914, 117 p.

DE LA ROCHE, Mazo, Québec. L'épopée du Canada, trad. Maurice Giguet, Paris, Ed. Begh, 1950, 338 p.

DESJARDINS, L.G., l'Angleterre, le Canada et la Grande Guerre, Québec, 1917, trans. England, Canada and the Great War, Québec, 1918, xvi-422 p.

DEVINE, Edward, les Jésuites martyrs de la Nouvelle-France, trad. The Canadian Martyrs, Paris, $1927,268 \mathrm{p}$

DION-LEVESQUE, Rosaire, Walt Whitman, ses meilleures pages, trad. par [...] Montréal, 1933, $240 \mathrm{p}$

DRUMMOND, Margaret-Mary, la Vénérable Mère Margerite Bourgeoys; sa vie et son temps par [...] trad. Joseph Bruneau, p.s.s., Montréal-Paris, s.d., xxv-251 p.

DUFFERIN, Lord, Un voyage en yacht, Lettres de hautes latitudes [...], trad. Benj. Sulte, Montréal, $1876, x v-320 \mathrm{p}$.

Lettres écrites des régions polaires, trad. F. de Lanoye, Paris, $1882,302 \mathrm{p}$. et gravures, portraits

DUGAS, G. (abbé), The Canadian West. Its Discovery... down the Year 1822, trans. from the French, Montréal, $1905,320 \mathrm{p}$

FERRES, James, The Chopping Bee and Other Laurentian Stories, by Marie Victorin, translated by [... ] Toronto, 1925, $256 \mathrm{p}$.

GREGORY, J.U., Récits de voyage en Floride, au Labrador, sur le fleuve Saint-Laurent, trad. A. Gagnon, Montréal, 1913, $244 \mathrm{p}$.

HOUDE, F., le Manoir mystérieux, 1913, trad. libre du Kenilworth de Walter Scott.

HUGHES, E.C., Rencontre de deux mondes... trad. J.C. Falardeau, Montréal, 1944, 388 p.

KALM, Peter, Voyage de Kalm en Amérique, analysé et trad., L.W. Marchand, Montréal, T. Berthiaume, 1880,2 vols, xvi-169, 257 p.

LAPALME, Robert, les Vingt Premières années du caricaturiste canadien - The First Twenty Years of Canadian Caricaturist, préf. Gratien Gélinas et intro. Jean-Louis Gagnon, Montréal, C.L.F., 1950 (texte et présentation bilingue).

LEMAY, L.P., Essais poétiques, Québec, 1865 , xii-310 p. (contient la 1 re éd. d'Évangéline de Longfellow en français), 2e éd., Québec, 1870, 192 p.; 3e éd., Montréal, 1912, 209 p.

LESPÉRANCE, John, les Bastonnais, Montréal, 1896 (trad. The Bastonnais, Toronto, 1877).

LEWIS, John, Mackenzie King, premier ministre... trad. D.-J. H. Montréal, 1929.

LONGFELLOW, H.W., Évangéline, conte d'Acadie, trad. avec intro, par Godefroid Kurth, Liège, $1883,98 \mathrm{p}$.

LUDWIG, Emil, Mackenzie King, esquisse... trad. André Champoux, Montréal, l'Arbre, 1944

MACKENZIE KING, W.-L., la Question sociale et le Canada, trad. Altiar, préf. G. Hanotaux, Paris, Alcan, 1925.

MAYNE-REID (Capt.), Bruin ou les chasseurs d'ours, trad. A. Letellier, Paris, 1905. les Exilés dans la forêt, trad. H. Loreau, Paris, 1901. Bruno ou les chasseurs d'ours, trad. M. Guerrier de Haupt, Tours, 1889.

Manuel abrégé de controverses ou Controverse des pauvres, trad. de l'anglais de J. Mannock, Québec, $1806,172 \mathrm{p}$

MAXINE, Unknown Fairies of Canada et Stowaways (A Tale of Old French Canada), Montréal, Granger

McDOWELL, F.D., la Route de Champlain, trad. Georges Panneton, Trois-Rivières-Québec, Macmillan-Bélisle, xix-492 p.

McLENNAN, Wm. Songs of Old Canada, tanslated, Montréal, 1886, $83 \mathrm{p}$

MORIN, Victor, les Ramezay et leur château-Ramezay Family and Chateau, trans. John D. King, Montréal, $1955,130 \mathrm{p}$

NAUD, Ogilvie, Sir J.J.C. Abbott, trad. Jos. Marmette, 1891 (tiré à part, 16 p.).

Sir Donald A. Smith, trad. Jos. Marmette, 1891 (tiré à part, 12 p.).

OWL, Grey, Un homme et ses bêtes, trad. Jeanne Rocke-Mazon, Paris, Boivin, 1937, xvi-318 p.

PAR KER, Gilbert, Femme ou sabre, trad. N. Levasseur, Québec, 1898, viii-289 p.

PARKMAN, Francis, la Prise de Québec et ses conséquences, trad. Ulric Barthe, Québec, 1908. ix-233 p. (Trad. d'une partie du Montcalm and Wolf). les Jésuites dans l'Amérique du Nord... Paris, 1882, $378 \mathrm{p}$.

POPE, Jos., Jacques Cartier. His Life and Voyages, trans. Jacques Cartier. Sa vie et ses voyages, Ottawa, 1890.

POULIOT, J.-Camille, Quebec and the Isle of Orleans, trans. J.-C. Paris-Québec, 1927, 244 p.

POUTRÉ, Félix, Escaped from the gallows... Montréal, 1885, (trad. de l'éd. française, Montréal, 1862)

SMITH, Goldwin, Devant le tribunal de l'histoire. Un plaidoyer... trad. Henri Bourassa, Montréal, 1903, $61 \mathrm{p}$. 
SULTE, B. and David, L.O., A History of Quebec, Montréal, 1908, 2 vols. (Le 2e vol. contient 265 biographies).

SWIFT, S.C./MARQUIS, T.G., The Voyages of Jacques Cartier, in prose and verse. Toronto, 1934, 79 p. - ill.

WOODS, W.C., The Isle of the Massacre following the French of the late J.C. Tache, Toronto, 1901, 98 p. SRONG, G.M. (ed.), Louisbourg in 1745, the Anonymous Lettre d'un habitant de Louisbourg, ed. with English trans., Toronto, 1897, $74 \mathrm{p}$. 